\title{
Seqüência de Möbius: resultados a longo prazo, da correção cirúrgica do estrabismo
}

\author{
Möbius sequence:long-term strabismus surgical outcome
}

\author{
Liana Oliveira Ventura ${ }^{1}$ \\ Cristiane Bezerra da $\mathrm{Cruz}^{2}$ \\ Henderson Celestino de Almeida ${ }^{3}$ \\ Marilyn Millar ${ }^{4}$ \\ Alessandra Freitas Carneiro Lira ${ }^{5}$ \\ Daniela Lyra Antunes ${ }^{6}$
}

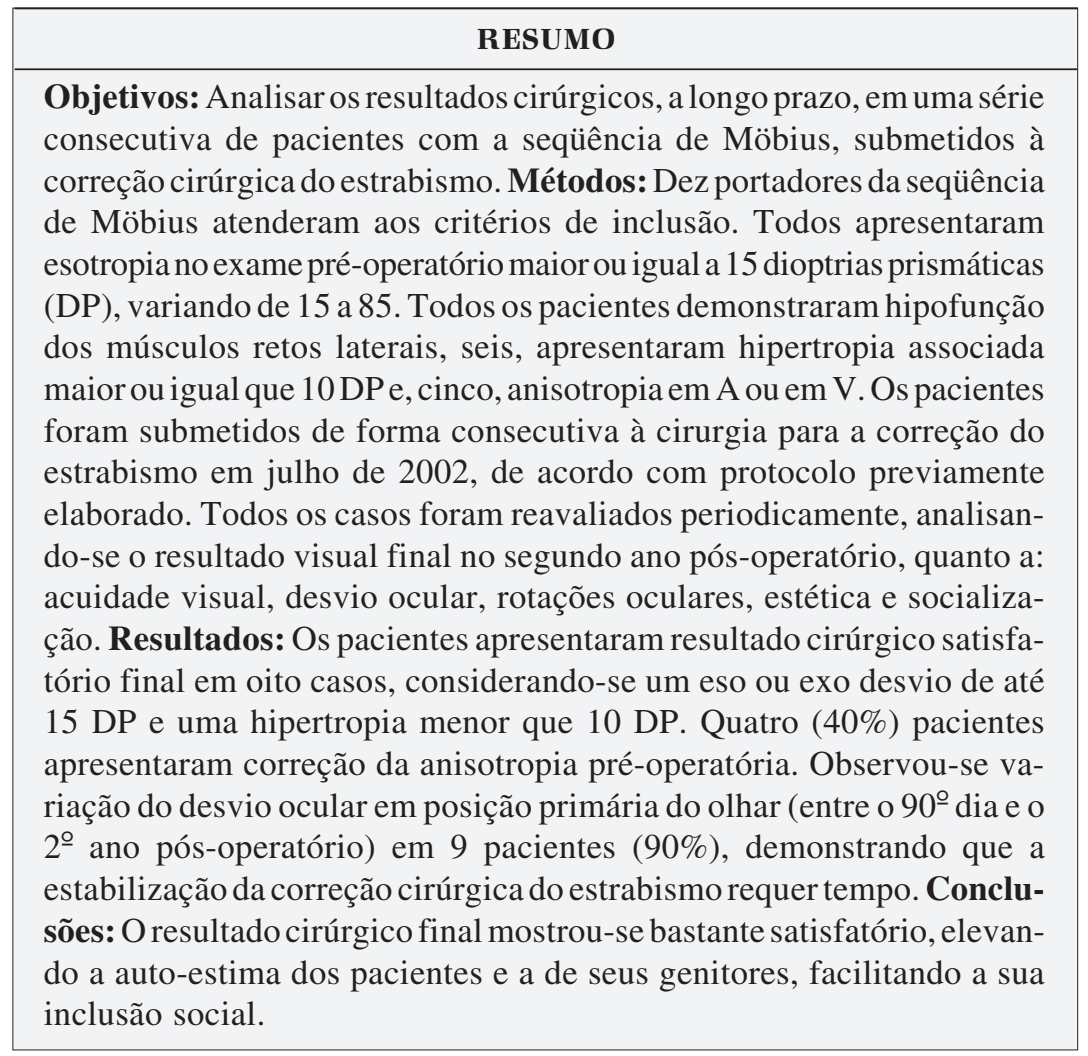

Descritores: Estrabismo/cirurgia; Síndrome de Möbius; Paralisia facial; Músculos oculomotores; Fixação ocular

\section{INTRODUÇÃO}

A seqüência de Möbius apresenta-se através da paresia ou paralisia congênita, uni ou bilateral, do $6^{\circ}$ e $7^{\circ}$ nervos cranianos. Freqüentemente associa-se a paresia/paralisia de outros nervos cranianos, anomalias craniofaciais e músculo-esqueléticas ${ }^{(1-2)}$. Pode estar associada com a síndrome de Poland $^{(3)}$ e ainda, com retardo mental e autismo ${ }^{(1,4-5)}$.

Vários fatores etiológicos têm sido relacionados à seqüência de Möbius e, embora existam casos de ocorrência familiar, alguns com alterações cromossômicas, a maioria é de aparecimento esporádico, sem herança mendeliana definida, sendo ambos os sexos afetados com igual freqüência ${ }^{(1,6)}$. $O$ mecanismo etiopatogênico mais aceito para a seqüência de Möbius seria uma insuficiência vascular no início da embriogênese, devido a agressões ambientais, como: coleta de vilosidade coriônica ${ }^{(1,7)}$; exposição a talidomida ${ }^{(7-9)}$; 
misoprostol $^{(8-11)}$; benzodiazepínicos ${ }^{(12)}$; cocaína ${ }^{(13)}$; álcool etílico ${ }^{(1)}$, além de choque elétrico, hipertermia e ruptura prematura de membranas ${ }^{(14)}$.

Os achados oculares mais freqüentes são o estrabismo convergente devido à disfunção dos abducentes e o lagoftalmo pelo acometimento dos nervos faciais ${ }^{(15)}$. A esotropia está presente em menos de $50 \%$ dos casos ${ }^{(16)}$. Os movimentos oculares verticais geralmente estão preservados. Entretanto, o envolvimento do $3^{\circ}$ nervo craniano é mais comum do que o suposto inicialmente.

O tratamento oftalmológico das crianças com a seqüência de Möbius deve ser feito principalmente quanto ao estrabismo e à exposição corneana, devido ao acometimento do $7^{\circ}$ nervo craniano ${ }^{(1)}$. Deve-se inicialmente prescrever a correção óptica e tratar a ambliopia; posteriormente, realiza-se a correção cirúrgica do estrabismo ${ }^{(17)}$.

Tendo em vista a importância da correção cirúrgica do estrabismo para os pacientes com a sequiência de Möbius, visando sua melhor inserção social e aceitação pelos familiares, o presente estudo foi realizado com o objetivo de analisar os resultados cirúrgicos a longo prazo, encontrados em uma série de pacientes com a seqüência de Möbius, submetidos à correção cirúrgica do estrabismo, de forma consecutiva.

\section{MÉTODOS}

Foram selecionados 10 casos de seqüência de Möbius que atendiam aos critérios de inclusão para o presente estudo - os pacientes apresentavam desvio ocular horizontal (esotropia) em posição primária do olhar (PPO) superior ou igual a 15 dioptrias prismáticas (DP). Oito pacientes apresentavam hipertropia associada, sendo que 6 apresentavam hipertropia maior ou igual a $10 \mathrm{DP}$. A anisotropia em $\mathrm{V}$ foi evidenciada em 4 casos e em A em 1 (Quadros 1 e 2).

Cinco pacientes eram do sexo feminino e 5 do masculino, com idade variando de 2 a 11 anos, média de 6 anos, desvio padrão de 2,8 anos. Todos foram submetidos à cirurgia para correção de estrabismo, de forma consecutiva, sob um mesmo protocolo previamente elaborado, em julho de 2002. Os pacientes foram analisados quanto a: acuidade visual; erro refrativo; ângulo de desvio medido pelo teste de cobertura com prismas ou pelo teste de Krimsky na posição primária do olhar, supra e infraversões e rotações oculares.

A acuidade visual para longe, com e sem correção óptica, foi medida de acordo com a idade e a colaboração do paciente. Foi avaliada monocularmente, através do olhar preferencial com cartões de acuidade visual de Teller em menores de 3 anos, LogMAR em portadores de visão subnormal ou pouco cooperativos e tabelas de Snellen nos maiores de 4 anos.

Nas rotações oculares, determinou-se hipofunção muscular variando numa escala de -1 a -8 . Classificou-se a hipofunção (dos músculos retos mediais e retos laterais) em leve quando -1 , moderada quando de -2 e -3 , severa quando -4 e de -5 a -8 quando além de hipofunção severa havia contratura muscular detectada através de ducção passiva pré-operatória.
Considerou-se anisotropia em A quando os pacientes apresentaram diferença no desvio ocular igual ou superior a 10 DP em medidas obtidas na supraversão e infraversão, e em $\mathrm{V}$ quando igual ou superior a $15 \mathrm{DP}$.

O procedimento cirúrgico consistiu no retrocesso dos músculos retos mediais nos pacientes com esotropia maior que $15 \mathrm{DP}$; os casos que apresentavam desvio vertical maior ou igual que $10 \mathrm{DP}$ associado foram submetidos ao retrocesso do reto superior. Aqueles com anisotropia em A foram submetidos a desinserção de oblíquos superiores e, os com anisotropia em V, ao deslocamento inferior dos retos mediais. Todos os pacientes foram examinados com três medidas prévias do estrabismo através do teste de cobertura ou método de Krimsky em todas as posições do olhar e análise das versões. Considerou-se um resultado cirúrgico satisfatório um eso ou exodesvio de até $15 \mathrm{DP}$ e uma hipertropia menor que $10 \mathrm{DP}$.

No pré-operatório, realizou-se biópsia dos músculos retos mediais operados para estudo histopatológico.

Os pacientes foram examinados no $1^{\circ}, 10^{\circ}, 45^{\circ}$ e $90^{\circ}$ dias pós-operatórios, de forma que os resultados fizeram parte de estudo preliminar ${ }^{(24)}$. Esses pacientes foram reexaminados no curso do $2^{\circ}$ ano de seguimento pós-operatório, seguindo o mesmo protocolo.

Este trabalho foi submetido à aprovação do Comitê de Ética e Pesquisa da Fundação Altino Ventura.

\section{RESULTADOS}

O procedimento cirúrgico realizado nos pacientes pode ser observado no quadro 1.

Os resultados cirúrgicos podem ser verificados no quadro 2. Observou-se variação do desvio ocular em PPO (entre o $90^{\circ}$ dia e o $2^{\circ}$ ano pós-operatórios) em 9 pacientes (90\%).

Através da análise de variância de medidas repetidas, as médias e desvios padrões das acuidades visuais dos olhos avaliados como variáveis contínuas foram respectivamente: Olho direito - pré-operatória $=0,62 \pm 0,27$, pós-operatória $(90$ dias $)=0,68 \pm 0,24$, pós-operatória $(2$ anos $)=0,68 \pm 0,29$; $\mathrm{p}=0,488$ e olho esquerdo - pré-operatória $=0,60 \pm 0,30$, pósoperatória $(90$ dias $)=0,61 \pm 0,27$, pós-operatória $(2$ anos $)=$ $0,60 \pm 0,30 ; \mathrm{p}=0,991$.

Quando se compararam os desvios pré-operatórios e pósoperatórios de 90 dias, observa-se que o resultado cirúrgico satisfatório nesse período foi de 7 casos $(70 \%)$. O resultado cirúrgico final, após 2 anos de seguimento revela resultado satisfatório em 8 casos $(80 \%), p=0,008$. As comparações foram realizadas pelo teste de McNemar. Um caso apresentava-se como satisfatório na avaliação pós-operatória de 90 dias evoluindo para insatisfatório na avaliação pós-operatória de 2 anos. Dois casos apresentavam-se como insatisfatórios na avaliação pós-operatória de 90 dias evoluindo para satisfatórios na avaliação pós-operatória de 2 anos.

Houve melhora da abdução em 7 casos (Quadro 3). 


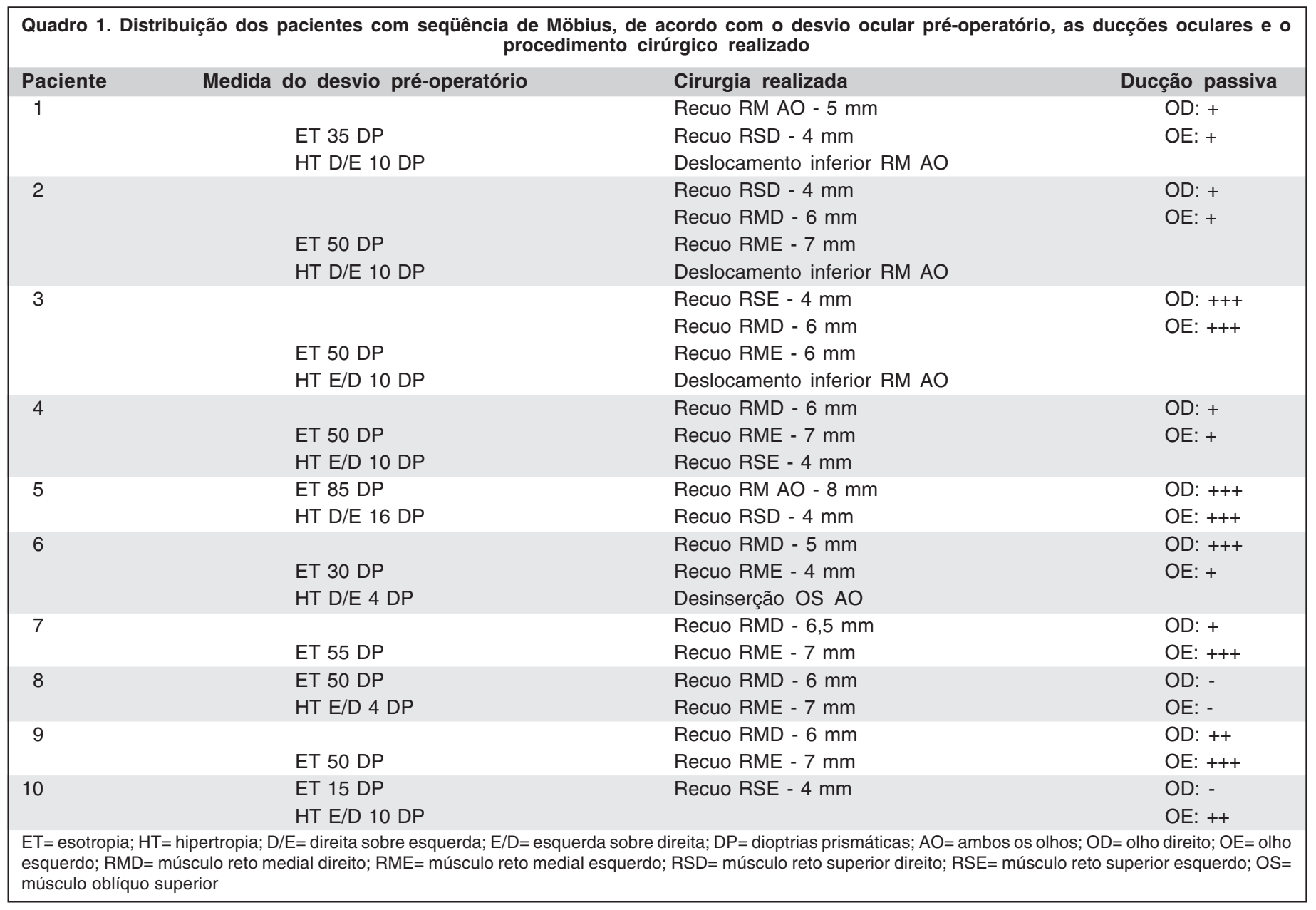

\begin{tabular}{|c|c|c|c|c|c|c|}
\hline \multirow{2}{*}{$\begin{array}{l}\text { Pact. } \\
\mathrm{N}^{\circ}\end{array}$} & \multicolumn{3}{|c|}{ Medida do desvio em posição primária do olhar } & \multicolumn{3}{|c|}{ Anisotropia alfabética } \\
\hline & Pré-operatório & $90^{\circ}$ dia & $2^{\circ}$ ano & Pré-operatório & $90^{\circ} \mathrm{dia}$ & $2^{\circ}$ ano \\
\hline 1 & $\begin{array}{l}\text { ET } 35 \text { DP } \\
\text { HT D/E } 10 \text { DP }\end{array}$ & ET 4 DP & ORTO & V & - & - \\
\hline 2 & $\begin{array}{l}\text { ET } 50 \text { DP } \\
\text { HT D/E } 10 \text { DP }\end{array}$ & ET $10 \mathrm{DP}$ & XT 6 DP & V & - & - \\
\hline 3 & $\begin{array}{l}\text { ET } 50 \text { DP } \\
\text { HT E/D } 10 \text { DP }\end{array}$ & XT 8 DP & $\begin{array}{l}\text { XT } 35 \text { DP } \\
\text { HT D/E } 4 \text { DP }\end{array}$ & $\mathrm{V}$ & - & $\mathrm{V}$ \\
\hline 4 & $\begin{array}{l}\text { ET } 50 \text { DP } \\
\text { HT E/D } 10 \text { DP }\end{array}$ & ET $10 \mathrm{DP}$ & $\mathrm{XT} 10 \mathrm{DP}$ & - & - & - \\
\hline 5 & $\begin{array}{l}\text { ET } 85 \text { DP } \\
\text { HT D/E } 16 \text { DP }\end{array}$ & ET 26 DP & ET 40 DP & - & - & - \\
\hline 6 & $\begin{array}{l}\text { ET } 30 \text { DP } \\
\text { HT D/E } 4 \text { DP }\end{array}$ & ORTO & ORTO & A & - & - \\
\hline 7 & ET 55 DP & HT E/D 4 DP & $\begin{array}{l}\text { XT } 6 \text { DP } \\
\text { HT E/D } 2 \text { DP }\end{array}$ & - & - & V \\
\hline 8 & $\begin{array}{l}\text { ET } 50 \text { DP } \\
\text { HT E/D } 4 \text { DP }\end{array}$ & ET 16 DP & ET 10 DP & - & - & - \\
\hline 9 & ET 50 DP & ORTO & ET 5 DP & - & V & V \\
\hline 10 & $\begin{array}{l}\text { ET } 15 \text { DP } \\
\text { HT E/D } 10 \text { DP }\end{array}$ & ET 16 DP & ET $10 \mathrm{DP}$ & V & - & - \\
\hline
\end{tabular}




\begin{tabular}{|c|c|c|c|c|c|c|}
\hline \multicolumn{7}{|c|}{$\begin{array}{l}\text { Quadro 3. Distribuição dos pacientes quanto às rotações oculares } \\
\text { pré e pós-operatórias de } 90 \text { dias e de } 2 \text { anos }\end{array}$} \\
\hline \multirow{2}{*}{$\begin{array}{l}\text { Pact. } \\
\mathrm{N}^{\circ}\end{array}$} & \multicolumn{2}{|c|}{ Pré-operatório } & \multicolumn{2}{|c|}{90 dias } & \multicolumn{2}{|c|}{2 anos } \\
\hline & OD & OE & OD & OE & OD & OE \\
\hline 1 & ${ }_{-6}^{N} \underset{N}{\chi_{N}^{N}}{ }^{N}-2$ & ${ }^{-1}{\underset{N}{N}}_{N}^{N}-3$ & ${ }_{-4}^{\stackrel{N}{\chi_{N}^{N}}}{ }_{N}^{N}$ & ${ }_{-1}^{N}{\underset{N}{N}}_{N}^{N}-4$ & ${ }_{-6}^{N}{\underset{N}{N}}^{\mathrm{N}}-2$ & ${ }_{-2}^{N} \overbrace{N}^{N}-4$ \\
\hline 2 & $-7{\underset{-1}{2}}_{-1}^{2}-1$ & ${ }^{2}{\underset{N}{N}}_{N}^{N}-7$ & ${ }_{-5}^{N}{\underset{N}{N}}^{N}-1$ & ${ }_{-2}^{N}{\underset{N}{N}}^{N}-4$ & ${ }^{-4} \underbrace{N}_{N}{ }_{N}^{N}-3$ & ${ }^{-4}{\underset{N}{N}}^{N}$ \\
\hline 3 & ${ }^{-4}{\underset{N}{N}}_{N}^{N}{ }^{N}$ & ${ }^{-1}{\underset{N}{N}}_{N}^{N}{ }^{N}$ & ${ }^{-3}{\underset{N}{N}}_{N}^{N}{ }^{N}$ & ${ }_{-2}^{N} \chi_{N}^{N}-3$ & ${ }^{-4}{\underset{N}{N}}_{N}^{N}-4$ & \\
\hline 4 & $\left.{ }^{-6} \underset{\mathrm{N}}{-1}\right\rangle_{\mathrm{N}}^{-1}-2$ & ${ }_{-2}^{\stackrel{N}{X} \boldsymbol{C}_{-1}^{N}-6}$ & ${ }_{-3}^{N}{\underset{N}{N}}_{N}^{N}-3$ & ${ }^{2}{\underset{N}{N}}_{N}^{N}-3$ & $-4 \underbrace{N}_{N}{ }_{N}^{N}-3$ & ${ }^{3}{\underset{N}{N}}^{N}$ \\
\hline 5 & ${ }_{-7}^{N} \underset{-1}{\chi_{-1}^{N}}$ & & ${ }_{-4}^{N}{\underset{N}{N}}^{N}-1$ & & ${ }^{-5}{\underset{N}{\mathrm{~N}}}_{\mathrm{N}}^{\mathrm{N}}-2$ & \\
\hline 6 & ${ }_{-5}^{N} \underset{N}{\chi_{N}}{ }^{1}-3$ & ${ }^{-3}{\underset{2}{2} \chi_{N}^{N}}^{\chi^{N}}-5$ & ${ }_{-4}^{N}{\underset{N}{N}}^{N}-2$ & ${ }_{-3}^{N} \underset{N}{\chi_{N}^{N}}-4$ & ${ }^{-4}{\underset{N}{N}}_{N}^{N}$ & \\
\hline 7 & ${ }^{-6}{\underset{N}{N}{ }_{N}^{N}}^{\chi_{N}^{N}}$ & ${ }^{-3}{\underset{N}{N}}_{N}^{N}-5$ & ${ }^{-4}{\underset{N}{N}}_{N}^{N}-4$ & ${ }_{-4}^{\stackrel{N}{\chi_{N}^{N}}-4}$ & ${ }^{-4} \underbrace{\mathrm{N}}_{\mathrm{N}}{\underset{\mathrm{N}}{\mathrm{X}}}^{\mathrm{N}}-4$ & $-4>$ \\
\hline 8 & $-5{\underset{N}{X}}_{N}^{-1}-3$ & $-2{\underset{N}{\mathrm{~N}}}^{\mathrm{N}}-5$ & $-4{\underset{N}{\mathrm{~N}}}_{\mathrm{N}^{\mathrm{N}}}^{\mathrm{N}}-2$ & ${ }^{2}{\underset{N}{N}}^{N}-5$ & $-4{\underset{N}{\mathrm{~N}}}^{\mathrm{N}}-3$ & $-2 \underbrace{N}_{N}{ }^{N}$ \\
\hline 9 & ${ }^{-6} \underset{\mathrm{N}}{-1} \mathrm{~N}^{-1}-1$ & ${ }_{-1}^{N}{\underset{N}{X}}^{\chi_{N}^{N}}-6$ & ${ }^{-4} \underbrace{N}_{N}{ }^{N}-1$ & ${ }_{-1}^{N}{\underset{N}{N}}_{N}^{N}-4$ & $-4{\underset{N}{N}}^{X_{N}^{N}}-3$ & ${ }^{-3} \underbrace{N}_{N}{ }^{N}$ \\
\hline 10 & $\underset{N}{-4} \underset{-1}{\chi_{-2}^{-2}}-2$ & ${ }^{2} \overbrace{1}^{N}-4$ & ${ }_{-4}^{N}{\underset{N}{N}}^{N}-1$ & ${ }_{-1}^{N}{\underset{N}{N}}^{N}-4$ & ${ }^{-4}{\underset{N}{N}}^{N}{ }^{N}$ & ${ }_{-3}^{N} \overbrace{N}^{N}$ \\
\hline
\end{tabular}

\section{DISCUSS ÃO}

O tratamento cirúrgico do esodesvio de pequeno ângulo em pacientes com seqüência de Möbius geralmente é contraindicado, pois se refere melhora espontânea do desvio em alguns $\operatorname{casos}^{(18)}$. Em nosso estudo, foi observada melhora espontânea do esodesvio em 1 caso, o qual havia sido submetido apenas à correção cirúrgica do desvio vertical.

Em estudo pré-operatório de 6 portadores da seqüência de Möbius, foi relatada inserção posteriorizada do reto medial em 2 casos, sendo que em 1 deles houve também dupla inserção deste músculo; o teste de ducção passiva foi negativo em 1 paciente e positivo no restante e a restrição mecânica foi proporcional à magnitude do desvio ${ }^{(2)}$. Na presente amostra, não se detectaram anomalias de inserção muscular. $O$ teste de ducção passiva foi negativo em 2 casos, e positivo nos demais, havendo proporcionalidade direta, entre esta e o esodesvio.

O procedimento de Hummelsheim foi relatado para correção cirúrgica em um caso de síndrome de Möbius, já operado sem obtenção de alinhamento ocular. Encontrou-se inserção posteriorizada dos retos mediais e aplasia bilateral de retos mediais e laterais. $\mathrm{O}$ reto medial direito era composto por extensa área de fibrose inserida anormalmente no globo. Estudos histopatológicos revelaram ausência de tecido muscular, assim como também foram encontradas alterações estruturais musculares nos pacientes do presente estudo, devido a provável disgenesia mesodérmica ${ }^{(16)}$.

Em relato de 1 paciente portador de seqüência de Möbius, operado com esodesvio alternante de 20 a 25 DP e anisotropia em A, foi obtida ortotropia após o retrocesso dos retos mediais e deslocamento muscular superior ${ }^{(19)}$. Nesse estudo, houve correção da anisotropia em A, através da desinserção dos músculos oblíquos superiores e da anisotropia em V com o retrocesso e deslocamento inferior dos músculos retos mediais, com exceção de 1 caso; 2 casos que não apresentavam anisotropia passaram a apresentá-la no período pós-operatório.

Ainda que a maior parte dos estrabismos tenha como causa um distúrbio do comando oculomotor, isto é, um fator relacionado ao sistema nervoso central, a correção objetiva mudar as posições, comprimentos e tensões dos músculos extra-oculares, para que suas respostas se tornem adequadas. Dependendo do tipo de procedimento cirúrgico realizado, o que se pode ter é uma previsibilidade quanto à correção, pois ela dependerá das readaptações dos estímulos inervacionais aos músculos ${ }^{(20)}$. Isso corrobora com os dados obtidos no presente estudo, em que houve variação do desvio em PPO em 9 pacientes quando se compara a avaliação do $90^{\circ}$ dia com a do $2^{\circ}$ ano pós-operatório, mostrando que a estabilidade do desvio é um processo que requer tempo, como já observado em outros estudos ${ }^{(20)}$.

Observou-se que a acuidade visual dos pacientes, no geral, permaneceu estável ao longo dos dois anos de evolução, apesar de que durante todo este período prescreveu-se o uso da correção óptica e oclusão nos casos com ambliopia. A pouca fidelidade ao tratamento anti-ambliopia destes pacientes justifica o resultado visual obtido, o que poderia ser atribuído ao baixo nível socioeconômico dos genitores. Na Fundação Altino Ventura, durante todo esse período, foram realizadas consultas periódicas, com prescrição e doação dos óculos. Porém, de acordo com o relatado pelos genitores, as crianças não deram seguimento ao tratamento de oclusão, apesar da insistência e orientação recebidas.

Verificou-se melhora do esodesvio em todos os pacientes, observando-se, no segundo ano, um resultado satisfatório em 8 casos $(80 \%)$, o que foi estatisticamente significante $(p=0,008)$, encorajando a submissão desses pacientes à cirurgia do estrabismo, quando adequadamente avaliados. A melhora da autoestima dos pacientes e genitores foi evidente em todos os casos. Sem dúvida alguma, a correção do estrabismo, tão evidente, e da expressão facial, representam a principal meta dos pais nesses casos.

\section{CONCLUSÕES}

Ocorreu resultado satisfatório em $80 \%$ dos casos, todavia, a variação do desvio ocular em PPO em $90 \%$ dos pacientes no período pós-operatório vem ratificar a afirmação de que a estabilidade do desvio é um processo que requer tempo. 
Ressalta-se que o resultado cirúrgico final dos pacientes mostrou-se bastante satisfatório, com boa situação estética em todos os casos, permitindo assim, a elevação da auto-estima dos pacientes e de seus genitores, facilitando a sua inclusão social e encorajando a submissão desses pacientes à cirurgia do estrabismo, quando adequadamente avaliados.

\section{ABSTRACT}

Purpose: To analyze the long-term results in a consecutive series of Möbius sequence patients, who underwent surgical correction of strabismus. Methods: Ten patients with Möbius sequence fulfilled the inclusion criteria of this study. All patients presented esotropia at the preoperative examination, above or equal to 15 prismatic diopters (DP) varying from 15 to 85 . All patients presented lateral rectus muscles severe underaction, six presented hipertropia above or equal $10 \mathrm{DP}$ associated with esodeviation, and five presented anisotropia in A or in V. The patients were operated upon protocol, in a consecutive way, in July 2002. Patients were reexamined periodically, and at $2^{\text {nd }}$ postoperative year as for: the visual acuity; deviation measurements; ocular rotations; cosmetic aspect and socialization. Results: The patients presented satisfactory surgical results in eight cases, considering an eso or exodeviation up to $15 \mathrm{DP}$ and a hipertropia lower than $10 \mathrm{DP}$. Four $(40 \%)$ patients presented correction of the preoperative anisotropia. Variation of ocular deviation in the primary position (from the $90^{\text {th }}$ day to the $2^{\text {nd }}$ postoperative year was observed) in 9 patients $(90 \%)$, demonstrating that strabismus surgical stabilization needs time. Conclusions: The surgical results were considered satisfactory, improving patient self-esteem and the parent satisfaction, making the social inclusion easier.

Keywords: Strabismus/surgery; Möbius syndrome; Facial paralysis; Oculomotor muscles; Fixation/ocular

\section{REFERÊNCIAS}

1. Miller MT, Stromland K. The Möbius sequence: a relook. J AAPOS. 1999;3(4): 199-208.
2. Vasconcelos GC, Silva FBD, Almeida HC de, Boas MLMV, Alvares MG. Síndrome de Moebius: achados clínicos e cirúrgico em sete pacientes. Arq Bras Oftalmol. 2001;64(3):211-5.

3. Kuklik M. Poland-Möbius syndrome and disruption spectrum affecting the face and extremities: a review paper and presentation of five cases. Acta Chir Plast. 2000;42(3):95-103.

4. Strömland K, Sjögreen L, Miller MT, Gilberg C, Wentz E, Johansson M, et al. Möbius sequence- a swedish multidiscipline study. Euro J Paediatr Neurol. 2002;6(1):35-45.

5. Costa AES, Ventura L, Almeida HC, Miller M, Lima RCR, Bandin JM. Seqüência de Moebius: aspectos sociais, psicológicos e cognitivos. An Fac Med Univ Fed Pernamb. 2001;46(2):137-43.

6. Borck G, Wirth J, Hardt T, Tonnies H, Brondum-Nielsen K, Bugge M, et al. Molecular cytogenetic characterization of a complex 46 , XY,t $(7 ; 8,11 ; 13)$ chromossome rearrangement in a patient with Moebius syndrome. J Med Genet. 2001;38(2):117-20.

7. Sromland K, Miller MT. Thalidomide embryopathy: revisited 27 years later. Acta Ophthalmol (Copenh). 1993;71(2):238-45.

8. Gonzalez CH, Marques-Dias MJ, Kim CA, Sugayama SMM, Da Paz JA, Huson SM, et al. Congenital abnormalities in Brazilian children associated with misoprostol misuse in first trimester of pregnancy. Lancet. 1998;351 (9116):1624-7. Comment in: Lancet. 1998;352(9124):323. Lancet. 1999;353 (9155):843-4.

9. Marques-Dia MJ. Fisiopatogenia da Síndrome de Moebius e da artrogripose correntes da exposição in utero ao misoprostol [tese]. São Paulo: Faculdade de Medicina da Universidade de São Paulo; 1999.

10. Boudoux DD, Matos MAG de, Gonçalves ED, Rocha M, Ventura LO, Hinrichsen SL. Síndrome de Moebius relacionada à ameaça de abortamento. Rev Bras Oftalmol. 2000;59(3):173-7.

11. Stillitano IG, Ventura LO, Miller MT, Almeida H, Tavares S. Sequiência de Möbius associada ao uso de misoprostol na gestação: detecção na maternidade. Rev Bras Oftalmol. 2001;60(11):812-6.

12. Courtens W, Vamos E, Hainaut M, Vergauwen P. Moebius syndrome in an infant exposed in utero to benzodiazepines. J Pediatr. 1992;121(5 pt 1):833-4. Comment on: J Pediatr. 1989;114(1):126-31.

13. Kankirawatana P, Tennison MB, D'Cruz OF, Greenwood RS. Moebius syndrome in infant exposed to cocaine in utero. Pediatr Neurol. 1993;9(1):71-2.

14. Fraco RG, Fraco PV, Fraco GK, Fraco AL, Fraco FM. Moebius syndrome: features and etiology. J Pediatr Ophthalmol Strabismus. 1988;35:304-11.

15. Henderson JL. The congenital facial diplegia syndrome: Clinical features, pathology and aetiology. A review of sixty-one cases. Brain. 1939;62:381-403.

16. Traboulosi EI, Maumenee IH. Extraocular muscle aplasia in Moebius syndrome. J Pediatr Ophthalmol Strabismus. 1986;23(3):120-2.

17. Almeida HC de, Ventura LO, Miller M, Reinaldo RE, Carneiro A. Seqüência de Möbius: resultados preliminares da correção cirúrgica do estrabismo. Rev Bras Oftalmol. 2004;63(9):464-72.

18. Miller MT, Ray V, Owens P, Chen F. Moebius and Moebius-like syndromes (TTV-OFM, OMLH). J Pediatr Ophthalmol Strabismus. 1989;26(4):176-88.

19. Watrhouse WJ, Enzenauer RW, Martiak AP. Successful strabismus surgery in a child with Moebius Syndrome. Ann Ophthalmol. 1993;25(8):292-4.

20. Foschini RMAS, Bicas HE. Cirurgias de músculos reto horizontais: análise de planejamentos e resultados. Arq Bras Oftalmol. 2001;64(6):523-34.

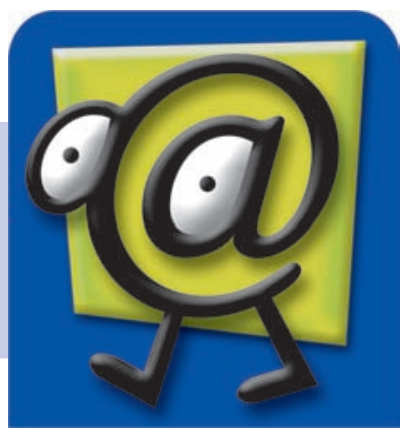

